\title{
A CHARACTERIZATION OF CERTAIN MEASURES USING QUASICONFORMAL MAPPINGS
}

\author{
CRAIG A. NOLDER
}

(Communicated by Clifford J. Earle, Jr.)

\begin{abstract}
Suppose that $\mu$ is a finite positive measure on the unit disk. Carleson showed that the $L^{2}(\mu)$-norm is bounded by the $H^{2}$-norm uniformly over the space of analytic functions on the unit disk if and only if $\mu$ is a Carleson measure. Analogues of this result exist for Bergmann spaces of analytic functions in the disk and in the unit ball of $C^{n}$. We prove here real variable analogues of certain Bergmann space results using quasiconformal and quasiregular mappings.
\end{abstract}

\subsection{INTRODUCTION}

We write $B^{n}(x, R)$ for the open ball in $n$-dimensional Euclidean space, $R^{n}$, centered at $x$ with radius $R$. We abbreviate $B^{n}=B^{n}(0,1)$. We write $m_{n}$ for the $n$-dimensional Lebesgue measure. For a set $E \subset R^{n}$ we write $|E|$ for $m_{n}(E)$. We denote the topological boundary of $E$ by $\partial E$. We assume throughout that $\Omega \subset R^{n}$ is open, $\partial \Omega \neq \varnothing$, and that $\mu$ is a positive finite measure on $\Omega$.

The following theorem is a special case of results found in $\left(\left[\mathrm{O}_{1}\right],\left[\mathrm{O}_{2}\right],[\mathrm{OP}]\right.$, $[\mathrm{H}],[\mathrm{S}],[\mathrm{CW}],[\mathrm{L}]$, and $[\mathrm{KS}])$.

1.2. Theorem. Suppose that $0<p \leq q<\infty$. If there exists a constant $C_{1}$ such that

$$
\mu\left(B \cap B^{2}\right) \leq C_{1} R^{2 q / p}
$$

for all balls $B=B^{2}(x, R)$ with $x \in \partial B^{2}$, then there exists a constant $C_{2}$, depending only on $p, q$ and $C_{1}$, such that

$$
\left(\int_{B^{2}}|f|^{q} d \mu\right)^{1 / q} \leq C_{2}\left(\int_{B^{2}}|f|^{p} d m_{2}\right)^{1 / p}
$$

for all analytic functions $f: B^{2} \rightarrow R^{2}$.

Received by the editors April 6, 1989.

1980 Mathematics Subject Classification (1985 Revision). Primary 30C60.

Key words and phrases. Carleson measures, norm inequalities, quasiregular mappings. 
Conversely, if (1.2) holds for all analytic functions $f: B^{2} \rightarrow R^{2}$, then (1.1) holds with $C_{1}$ depending only on $p, q$ and $C_{2}$.

Hastings ([H], p. 237) gives an analogue of Theorem 1.2 for plurisubharmonic functions on the unit polydisk in $C^{n}$. Stegenga ([S], p. 116, Theorem 1.2) generalizes Theorem 1.2, in the case that $p=q=2$, to include certain weights on the right-hand side of (1.2). Cima and Wogen, also when $p=q=2$, ([CW], p. 158, Theorem 1) give analogues of some of the results in [S] for holomorphic functions on the unit ball in $C^{n}$. Kerman and Sawyer [KS] give more general weighted results for analytic functions in the unit disk. See also Luecking [L] in the complex case.

The main result of this paper is the following real variable analogue of Theorem 1.2. Weighted results corresponding to Theorem 1.3 will be dealt with in another paper.

1.3. Theorem. Suppose that $0<p \leq q<\infty$. If there exists a constant, $C_{1}$, such that

$$
\mu\left(B \cap B^{n}\right) \leq C_{1} R^{n q / p}
$$

for all balls $B=B^{n}(x, R)$ with $x \in \partial B^{n}$, then there is a constant, $C_{2}$, depending on $p, q, n, K$, and $C_{1}$, such that

$$
\left(\int_{B^{n}}|f|^{q} d \mu\right)^{1 / q} \leq C_{2}\left(\int_{B^{n}}|f|^{p} d m_{n}\right)^{1 / p}
$$

for all $K$-quasiregular mappings $f: B^{n} \rightarrow R^{n}$.

Conversely, if (1.4) holds for all $K(n, p)$-quasiconformal mappings $f: B \rightarrow$ $R^{n}$, then (1.3) holds with $C_{1}$ depending on $p, q, n$, and $C_{2}$. Here $K(n, p)=$ $\max (2 n / p, p / 2 n)^{n-1}$.

Quasiregular and quasiconformal mappings are important generalizations of analytic functions and conformal mappings. We define these concepts in $\S 2$. In $\S 3$ we prove that (1.4) implies (1.3) and in $\S 4$ that (1.3) implies (1.4). In $\S 5$ we present an example which shows that (1.3) need not imply (1.4) in the case $q<p$.

In the case that $n=2$ and $K=1$, Theorem 1.3 reduces to Theorem 1.2.

We next give some corollaries.

1.4. Corollary. There is a constant $C$, depending only on $p, q, n$, and $K$, such that

$$
\left(\int_{B_{k}}|f(x)|^{q}(1-|x|)^{n q / p-k} d m_{k}\right)^{1 / q} \leq C\left(\int_{B^{n}}|f|^{p} d m_{n}\right)^{1 / p}
$$

for all $K$-quasiregular mappings $f: B^{n} \rightarrow R^{n}$. Here $k=1,2, \ldots, n, 0<p \leq$ $q<\infty, B_{k}=\left\{\left(x_{1}, x_{2}, \ldots, x_{n}\right) \in B^{n} \mid x_{j}=0, j=k+1, k+2, \ldots, n\right\}$ for $k=1, \ldots, n-1$ and $B_{n}=B^{n}$. 
1.5. Corollary. There is a constant $C$, depending only on $p, q, n$, and $K$, such that

$$
\left(\sum_{k=0}^{\infty}\left|f\left(\left(1-2^{-k}\right) S_{k}\right)\right|^{q} 2^{-n q k / p}\right)^{1 / q} \leq C\left(\int_{B^{n}}|f|^{p} d m_{n}\right)^{1 / p}
$$

for all $K$-quasiregular mappings $f: B^{n} \rightarrow R^{n}$. Here $0<p \leq q<\infty$ and $S_{k} \in \partial B^{n}, k=0,1,2, \ldots$.

1.6. Corollary. There is a constant $C$, depending only on $p, n$, and $K$, such that

$$
|f(x)| \leq C(1-|x|)^{-n / p}\left(\int_{B^{n}}|f|^{p} d m_{n}\right)^{1 / p}
$$

for all $K$-quasiregular mappings $f: B^{n} \rightarrow R^{n}$ and all $x \in B^{n}$.

\subsection{QUASIREgUlaR MAPPINGS}

We write $C^{\infty}(\Omega)$ for the space of functions having derivatives of all orders in $\Omega$. The Sobolev space, $W_{p}^{1}(\Omega), 1 \leq p<\infty$, is the completion of $C^{\infty}(\Omega)$ with respect to the norm

$$
\left(\int_{\Omega}|f|^{p} d m_{n}\right)^{1 / p}+\left(\int_{\Omega}|\nabla f|^{p} d m_{n}\right)^{1 / p}
$$

We write $W_{p, l o c}^{1}(\Omega)$ for $\cap W_{p}^{1}\left(\Omega^{\prime}\right)$ where the intersection is over all $\Omega^{\prime}$ compactly contained in $\Omega$. Here $D f$ is the derivative of $f,|D f|$ is the norm of $D f$ as a linear transformation, and $J_{f}=\operatorname{det} D f$.

2.2. Definition. A function $f: \Omega \rightarrow R^{n}, \Omega \subset R^{n}$, is $K$-quasiregular in $\Omega$, $1 \leq K<\infty$, if

$$
f \in W_{n, l o c}^{1}(\Omega)
$$

and if

$$
|D f|^{n} \leq K J_{f}
$$

almost everywhere in $\Omega$ with respect to $m_{n}$.

A nonconstant quasiregular mapping is continuous, open and discrete. When $n=2, f$ is 1-quasiregular if and only if it is an analytic function. A $K$ quasiregular mapping in $R^{n}$ is quasiconformal, in the usual sense, if and only if it is a homeomorphism. For further information on quasiregular mappings, see ([BI], [ $\left.\mathrm{MRV}_{1}\right],\left[\mathrm{MRV}_{2}\right],[\mathrm{R}]$, and [V]). The next result is given in [IN] (p. 279 , Proposition 3 ). Its proof involves improving exponents associated with reverse Hölder inequalities for quasiregular mappings. 
2.3. Theorem. If $f$ is $K$-quasiregular in $\Omega$, then

$$
|f(x)| \leq C\left(\frac{1}{|Q|} \int_{Q}|f|^{r} d m_{n}\right)^{1 / r}
$$

for each cube $Q$ with $\bar{Q} \subset \Omega$. Here $x$ is the center of $Q, 0<r<\infty$, and $C$ is a constant which depends only on $n, r$, and $K$.

\subsection{Proof that (1.4) implies (1.3)}

Let $\xi \in \partial B$ and $B=B^{n}(\xi, R)$ with $R<\frac{1}{2}$. We write $a=(1-R) \xi$ and $a^{*}=a /|a|^{2}$. Define a $K(n, p)$-quasiconformal mapping in $B^{n}$ as follows:

$$
f(x)=\left(x-a^{*}\right)\left|x-a^{*}\right|^{-(2 n+p) / p} \text {. }
$$

If $x \in B \cap B^{n}$, then

$$
(3(1-|a|))^{-2 n q / p} \leq|f(x)|^{q} .
$$

Using (3.1) and (1.4),

$$
\begin{aligned}
\mu\left(B \cap B^{n}\right) & \leq C_{3}(1-|a|)^{2 n q / p} \int_{B \cap B^{n}}|f|^{q} d \mu \\
& \leq C_{4}(1-|a|)^{2 n q / p}\left(\int_{B^{n}}|f|^{p} d m_{n}\right)^{q / p} \\
& \leq C_{5}(1-|a|)^{n q / p} .
\end{aligned}
$$

Hence $\mu$ satisfies (1.3) with

$$
C_{1} \leq \max \left(C_{5}, \mu\left(B^{n}\right) 2^{n q / p}\right) .
$$

\subsection{Proof that (1.3) IMPlies (1.4)}

We first prove the following local result which applies to functions which satisfy a certain sub-mean-value property with respect to cubes.

4.2. Theorem. Let $p, q>0$. Suppose that $f$ is measurable in $\Omega$ and suppose that there exists a constant $C_{1}$ such that

$$
\left|f\left(x_{Q}\right)\right| \leq C_{1}\left(\frac{1}{|Q|} \int_{Q}|f|^{p} d m_{n}\right)^{1 / p}
$$

for all cubes $Q$ with $\bar{Q} \subset \Omega$. Here $x_{Q}$ is the center of $Q$. If $Q_{0}$ is a cube with $\sigma Q_{0} \subset \Omega, \sigma>1$, and if there is a constant, $C_{2}$, such that

$$
\mu\left(Q_{0}\right) \leq C_{2}\left|Q_{0}\right|^{q / p},
$$

then there is a constant $C_{3}$, depending only on $p, q, n, \sigma, C_{1}$, and $C_{2}$, such that

$$
\left(\int_{Q_{0}}|f|^{q} d \mu\right)^{1 / q} \leq C_{3}\left(\int_{\sigma Q_{0}}|f|^{p} d m_{n}\right)^{1 / p} .
$$


Proof. Let $x_{0} \in Q_{0}$ and let $P$ be the cube centered at $x_{0}$ with side length equal to $\sigma-1$ times the sidelength of $Q_{0}$. Then $P \subset \sigma Q_{0}$ and by (4.1) we have that

$$
\begin{aligned}
\left|f\left(x_{0}\right)\right| & \leq C_{1}\left(\frac{1}{|P|} \int_{P}|f|^{p} d m_{n}\right)^{1 / p} \\
& \leq C_{1}\left(\frac{\sigma^{n}}{(1-\sigma)^{n}\left|\sigma Q_{0}\right|} \int_{\sigma Q_{0}}|f|^{p} d m_{n}\right)^{1 / p} .
\end{aligned}
$$

This is a uniform estimate at each point $x_{0} \in Q_{0}$ and we have

$$
\left(\int_{Q_{0}}|f|^{q} d \mu\right)^{1 / q} \leq C_{4} \mu\left(Q_{0}\right)^{1 / q}\left(\frac{1}{\left|\sigma Q_{0}\right|} \int_{\sigma Q_{0}}|f|^{p} d m_{n}\right)^{1 / p} .
$$

By combining (4.2) with (4.4) we obtain (4.3).

By the mean-value property and Hölder's inequality, (4.1) is satisfied for harmonic functions for $p \geq 1$. It also holds for harmonic functions when $0<p<\infty$ ([G], p. 121, Lemma 3.7). Quasiregular mappings satisfy condition (4.1) by Theorem 2.3. Hence to verify that (1.3) implies (1.4) we need only show that the weak local estimates (4.3) imply the global result. We actually prove the following more general result.

4.3. Theorem. Suppose that there is a constant $C_{1}$ such that

$$
\mu(B \cap \Omega) \leq C_{1} R^{n q / p}
$$

for all balls $B=B^{n}(\xi, R)$ with $\xi \in \partial \Omega$. If $0<p \leq q<\infty$, then there is a constant $C_{2}$, depending only on $n, p, q, K$, and $C_{1}$, such that

$$
\left(\int_{\Omega}|f|^{q} d \mu\right)^{1 / q} \leq C_{2}\left(\int_{\Omega}|f|^{p} d m_{n}\right)^{1 / p}
$$

for all $K$-quasiregular $f: \Omega \rightarrow R^{n}$.

Proof. We write diam $Q$ for the Euclidean diameter of the cube $Q, d(F, E)$ the Euclidean distance between sets $F$ and $E$, and $d(x, E)$ for $d(\{x\}, E)$. We consider a collection of open cubes $\left\{Q_{i}\right\}$ contained in $\Omega$ with the following properties:

$$
\begin{gathered}
\Omega=\bigcup_{i=1}^{\infty} \bar{Q}_{i}, \\
Q_{i} \cap Q_{j}=\varnothing \quad \text { for all } i \neq j, \\
\operatorname{diam}\left(Q_{i}\right) \leq d\left(Q_{i}, \partial \Omega\right) \\
\leq 4 \operatorname{diam}\left(Q_{i}\right)
\end{gathered}
$$

for all $i=1,2, \ldots$, and

$$
\text { if } \bar{Q}_{i} \cap \bar{Q}_{j} \neq \varnothing \text {, }
$$


then

$$
\begin{aligned}
\frac{1}{4} \operatorname{diam}\left(Q_{j}\right) & \leq \operatorname{diam}\left(Q_{i}\right) \\
& \leq 4 \operatorname{diam}\left(Q_{j}\right)
\end{aligned}
$$

for all $i, j=1,2, \ldots$.

Every $\Omega$ has such a cover which is often called a Whitney cover ([St], p. 16, Theorem 3). It follows from the above properties that

$$
\sum_{Q \in W} \chi_{\frac{5}{4} Q}(x) \leq 6^{n}
$$

where $\chi_{Q}$ is the characteristic function of $Q$.

Next for a given $Q \in W$, choose $x_{0} \in \partial \Omega$ such that

$$
d\left(x_{0}, \sqrt{\frac{5}{4}} Q\right)=d\left(\sqrt{\frac{5}{4}} Q, \partial \Omega\right) .
$$

It follows that $\sqrt{\frac{5}{4}} Q \subset B^{n}\left(x_{0}, \delta\right)$ where $\delta=d\left(x_{0}, \sqrt{\frac{5}{4}} Q\right)+\operatorname{diam}\left(\sqrt{\frac{5}{4}} Q\right)$. Hence

$$
\begin{aligned}
\mu\left(\sqrt{\frac{5}{4}} Q\right) & \leq \mu\left(B^{n}\left(x_{0}, \delta\right)\right) \\
& \leq C_{1} \delta^{n q / p} \\
& \leq C_{3}(\operatorname{diam} Q)^{n q / p} .
\end{aligned}
$$

By Theorem 4.2 it follows that

$$
\left(\int_{\sqrt{\frac{5}{4}} Q}|f|^{q} d \mu\right)^{1 / q} \leq C_{4}\left(\int_{\frac{5}{4} Q}|f|^{p} d m_{n}\right)^{1 / p} .
$$

Next we use the inequality

$$
\left(\sum_{k} t_{k}\right)^{\alpha} \leq \sum_{k} t_{k}^{\alpha}
$$

where $t_{k} \geq 0$ and $\alpha \leq 1$. Combining (4.7), (4.13) with $\alpha=p / q,(4.12)$ and (4.11) we have

$$
\begin{aligned}
\left(\int_{\Omega}|f|^{q} d \mu\right)^{p / q} & \leq\left(\sum_{Q \in W} \int_{\sqrt{\frac{5}{4}} Q}|f|^{q} d \mu\right)^{p / q} \\
& \leq \sum_{Q \in W}\left(\int_{\sqrt{\frac{5}{4}} Q}|f|^{q} d \mu\right)^{p / q} \\
& \leq C_{4}^{p} \sum_{Q \in W} \int_{\frac{5}{4} Q}|f|^{p} d m_{n} \\
& \leq 6^{n} C_{4}^{p} \int_{\Omega}|f|^{p} d m_{n} .
\end{aligned}
$$




\section{AN EXAMPLE WHEN $q<p$}

Let $e_{1}=(1,0, \ldots, 0)$ and define

$$
f_{\beta}(x)=\left(x-e_{1}\right)\left|x-e_{1}\right|^{\beta-1}
$$

where $-n / p<\beta<-n / 2 p$. Each $f_{\beta}(x)$ is $K$-quasiconformal with $K=$ $\max (n / p, 2 p / n)^{n-1}$. Consider

$$
\begin{aligned}
\int_{B^{n}}\left|f_{\beta}(x)\right|^{p} d m_{n} & \leq \int_{B\left(e_{1}, 2\right)}\left|x-e_{1}\right|^{p \beta} d m_{n} \\
& =\frac{C(n) 2^{n+p \beta}}{n+p \beta} .
\end{aligned}
$$

Next let $x_{k}=\left(1-2^{-k}, 0, \ldots, 0\right), k=0,1,2, \ldots$, and define $\mu(E)=$ $\sum_{x_{k} \in E} 2^{-k \alpha}$ for $E \subseteq B^{n}$ with $\alpha=n q / p$. It follows that $\mu$ satisfies (4.5). Furthermore

$$
\begin{aligned}
\int_{B^{n}}\left|f_{\beta}\right|^{q} d \mu & =\sum_{k=0}^{\infty}\left|f_{\beta}\left(x_{k}\right)\right|^{q} 2^{-k \alpha} \\
& =\sum_{k=0}^{\infty} 2^{-k(q \beta+\alpha)} \\
& =\left(1-2^{-q(\beta+n / p)}\right)^{-1} .
\end{aligned}
$$

From (5.1) and (5.2) it follows that when $q<p$

$$
\begin{aligned}
\lim _{\beta \rightarrow-n / p^{+}} \frac{\left(\int_{B^{n}}\left|f_{\beta}\right|^{q} d \mu\right)^{p}}{\left(\int_{B^{n}}\left|f_{\beta}\right|^{p} d m_{n}\right)^{q}} & \geq \lim _{\beta \rightarrow-n / p^{+}} \frac{(n+p \beta)^{q}}{C(n)^{q} 2^{q(n+p \beta)}\left(1-2^{-q(n+p \beta) / p}\right)^{p}} \\
& \geq \lim _{\beta \rightarrow-n / p^{+}}\left(\frac{p}{2 C(n) q \log 2}\right)^{q}\left(1-2^{-q(n+p \beta) / p}\right)^{q-p} \\
& =\infty .
\end{aligned}
$$

\section{REFERENCES}

[BI] B. Bojarski and T. Iwaniec, Analytical foundations of the theory of quasiconformal mappings in $R^{n}$, Ann. Acad. Sci. Fenn. Ser. AI Math. 8 (1984), 257-324.

[CW] J. A. Cima and W. R. Wogen, A Carleson measure theorem for the Bergman space on the ball, J. Operator Theory 7 (1982), 157-165.

[G] J. B. Garnett, Bounded analytic functions, Academic Press, New York, 1981.

[H] W. W. Hastings, A Carleson measure theorem for Bergman spaces, Proc. Amer. Math. Soc. 52 (1975), 237-241.

[IN] T. Iwaniec and C. A. Nolder, Hardy-Littlewood inequality for quasiregular mappings in certain domains in $R^{n}$, Ann. Acad. Sci. Fenn. Ser. AI Math. 10 (1985), 267-282.

[KS] R. Kerman and E. Sawyer, Carleson measure and multipliers of Dirichlet-type spaces, Trans. Amer. Math. Soc. 309 (1988), 87-98.

[L] D. L. Luecking, A technique for characterizing Carleson measures on Bergman spaces, Proc. Amer. Math. Soc. 87 (1983), 656-660. 
[MRV $\left.{ }_{1}\right]$ O. Martio, S. Rickman, and J. Väisälä, Definitions for quasiregular mappings, Ann. Acad. Sci. Fenn. Ser. AI Math. 448 (1969), 1-40.

$\left[\mathrm{MRV}_{2}\right] \ldots$, Topological and metric properties of quasiregular mappings, Ann. Acad. Sci. Fenn. Ser. AI Math. 488 (1971), 1-31.

$\left[\mathrm{O}_{1}\right]$ V. L. Oleinik, Embedding theorems for weighted classes of harmonic and analytic functions, Zap. Nauchn. Sem. Leningrad. Otdel. Mat. Inst. Steklov. 47 (1974), 120-137.

$\left[\mathrm{O}_{2}\right] \ldots$ Embedding theorems for weighted classes of harmonic and analytic functions, J. Soviet Math. 9 (1978), 228-243.

[OP] V. L. Oleinik and B. S. Pavlov, Embedding theorems for weighted classes of harmonic and analytic functions, Zap. Nauchn. Sem. Leningrad. Otdel. Mat. Inst. Steklov. 22 (1971), 94-102.

[R] J. G. Rešetnjak, Estimates for the modulus of continuity for certain mappings, Sibirsk. Mat. Zh. 7 (1966), 1106-1114.

[S] D. A. Stegenga, Multiplies of the Dirichlet space, Illinois J. Math. 24 (1980), 113-139.

[St] E. M. Stein, Singular integrals and differentiability properties of functions, Princeton Univ. Press, Princeton, NJ, 1970.

[V] M. Vuorinen, Conformal geometry and quasiregular mappings, Springer-Verlag, New York, 1988.

Department of Mathematics, Florida State University, Tallahassee, Florida 323063027 